\title{
A Low-Cost, Smart Capacitive Position Sensor
}

\author{
Ferry N. Toth and Gerard C. M. Meijer
}

\begin{abstract}
A new high-performance, low-cost, capacitive position-measuring system is described. By using a highly linear oscillator, shielding and a three-signal approach, most of the errors are eliminated. The accuracy amounts to $1 \mu \mathrm{m}$ over a 1 $\mathrm{mm}$ range. Since the output of the oscillator can directly be connected to a microcontroller, an $A / D$ converter is not needed.
\end{abstract}

\section{INTRODUCTION}

$T^{4}$ HIS paper describes a novel high-performance, lowcost, capacitive displacement measuring system featuring:

- $1 \mathrm{~mm}$ measuring range,

- $1 \mu \mathrm{m}$ accuracy,

- $0.1 \mathrm{~s}$ total measuring time.

Translated to the capacitive domain, the specifications correspond to:

- a possible range of $1 \mathrm{pF}$; only $50 \mathrm{fF}$ of this range is used for the displacement transducer;

- $50 \mathrm{aF}$ absolute capacitance-measuring inaccuracy.

Meijer and Schrier [1] and more recently Van Drecht, Meijer, and De Jong [2] have proposed a displacementmeasuring system, using a PSD (Position Sensitive Detector) as sensing element. Some disadvantages of using a PSD are the higher costs and the higher power consumption of the PSD and LED (Light-Emitting Diode) as compared to the capacitive sensor elements described in this paper.

The signal processor uses the concepts presented in [2], but is adopted for the use of capacitive elements. By the extensive use of shielding, guarding and smart $A / D$ conversion, the system is able to combine a high accuracy with a very low cost-price. The transducer produces threeperiod-modulated signals which can be selected and directly read out by a microcontroller. The microcontroller, in return, calculates the displacement and can send this value to a host computer (Fig. 1) or a display or drive an actuator.

\section{The Electrode Structure}

The basic sensing element consists of two simple electrodes with capacitance $C_{x}$ (Fig. 2). The smaller one ( $\left.E_{2}\right)$ is surrounded by a guard electrode. Thanks to the use of

Manuscript received May 14, 1992; revised September 14, 1992. This work was supported by Delft Instruments Tank Gauging.

The authors are with the Department of Electrical Engineering, Delf University of Technology, Mekelweg 4, 2628 CD Delft, The Netherlands.

IEEE Log Number 9205053.

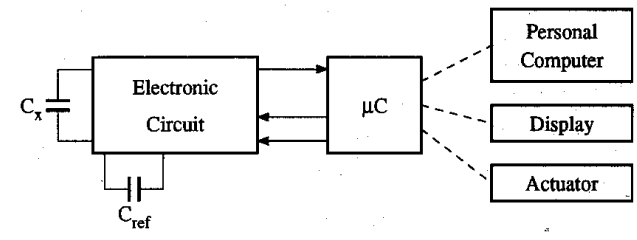

Fig. 1: Block diagram of the system.

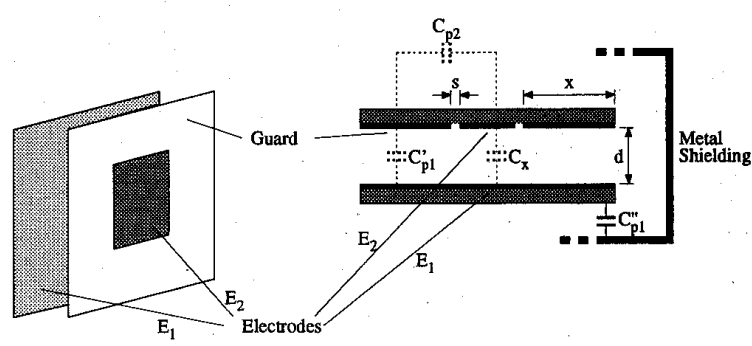

Fig. 2. Perspective and dimensions of the electrode structure.

the guard electrode, the capacitance $C_{x}$ between the two electrodes is independent of movements (lateral displacements as well as rotations) parallel to the electrode surface. The influence of the parasitic capacitances $C_{p}$ will be eliminated as will be discussed in Section III.

According to Heerens [3], the relative deviation in the capacitance $C_{x}$ between the two electrodes caused by the finite guard electrode size is smaller than:

$$
\delta<e^{-\pi(x / d)}
$$

where $x$ is the width of the guard and $d$ the distance between the electrodes. This deviation introduces a nonlinearity. Therefore we require that $\delta$ is less than $100 \mathrm{ppm}$. Also the gap between the small electrode and the surrounding guard causes a deviation:

$$
\delta<e^{-\pi(d / s)}
$$

with $s$ the width of the gap. This deviation is negligible compared to (1), when the gap width is less than $\frac{1}{3}$ of the distance between the electrodes.

Another cause of errors originates from a possible finite skew angle $\alpha$ between the two electrodes (Fig. 3). Assuming the following conditions:

- the potentials on the small electrode and the guard electrode are equal to $0 \mathrm{~V}$

- the potential on the large electrode is equal to $V$ volt,

- the guard electrode is large enough,

it can be seen that the electric field will be concentric. 


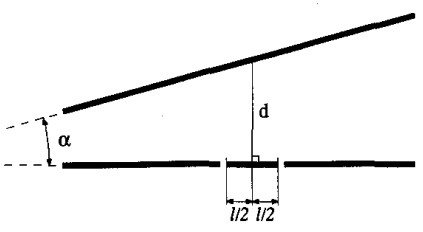

Fig. 3. Electrodes with angle $\alpha$.

To keep the calculations simple, we will assume the electrodes to be infinitely large in one direction. Now the problem is a two-dimensional one that can be solved by using polar-coordinates $(r, \varphi)$. In this case the electrical field can be described by:

$$
\vec{E}=\left(\begin{array}{c}
-\frac{V \sin \varphi}{\alpha r} \\
\frac{V \cos \varphi}{\alpha r}
\end{array}\right)
$$

To calculate the charge on the small electrode, we set $\varphi$ to 0 and integrate over $r$ :

$$
Q=\epsilon_{0} \int_{B_{l}}^{B_{r}} \frac{V}{\alpha r} d r
$$

with $B_{l}$ the left border of the small electrode:

$$
B_{l}=\frac{d}{\tan \alpha}-\frac{l}{2}
$$

and $B_{r}$ the right border:

$$
B_{r}=\frac{d}{\tan \alpha}+\frac{l}{2} .
$$

Solving (4) results in:

$$
Q=V \frac{\epsilon_{0}}{a} \ln \left(\frac{2 d \cos \alpha+l \sin \alpha}{2 d \cos \alpha-l \sin \alpha}\right) .
$$

For small $\alpha$ 's this can be approximated by:

$$
C=\frac{\epsilon_{0} l}{d}\left(1+\frac{4 d^{2}+l^{2}}{12 d^{2}} \alpha^{2}\right) .
$$

It appears to be desirable to choose $l$ smaller than $d$, so the error will depend only on the angle $\alpha$. In our case, a change in the angle of $0.6^{\circ}$ will cause an error less than $100 \mathrm{ppm}$.

With a proper design the parameters $\epsilon_{0}$ and $l$ are constant, and then the capacitance between the two electrodes will depend only on the distance $d$ between the electrodes.

\section{Elimination of Parasitic Capacitances}

Besides the desired sensor capacitance $C_{x}$ there are also many parasitic capacitances in the actual structure (Fig. 2). These capacitances can be modeled as shown in Fig. 4. Here $C_{p 1}$ represents the parasitic capacitances from the electrode $E_{1}$ and $C_{p 2}$ from the electrode $E_{2}$ to the guard electrodes and the shielding. Parasitic capacitance $C_{p 3}$ results from imperfect shielding and forms an offset capac-

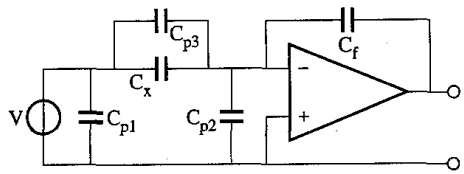

Fig. 4. Elimination of parasitic capacitances.

itance. When the transducer capacitance $C_{x}$ is connected to an $\mathrm{AC}$ voltage source and the current through the electrode is measured, $C_{p 1}$ and $C_{p 2}$ will be eliminated. $C_{p 3}$ can be eliminated by performing an offset measurement.

The current is measured by the amplifier with shunt feedback, which has a very low input impedance. To obtain the required linearity, the unity-gain bandwidth $f_{T}$ of the amplifier has to satisfy the following condition:

$$
f_{T}>\frac{12}{\pi T \frac{C_{f}}{C_{f}+C_{p 2}}}
$$

where $T$ is the period of the input signal.

Since $C_{p 2}$ consists of cable capacitances and the input capacitance of the op amp, it may indeed be larger than $C_{f}$ and can not be neglected.

\section{The Concept of the System}

The system uses the three-signal concept presented in [2], which is based on the following principles. When we measure a capacitor $C_{x}$ with a linear system, we obtain a value:

$$
M_{x}=m C_{x}+M_{\mathrm{off}}
$$

where $m$ is the unknown gain and $M_{\text {off }}$ the unknown offset. By performing the measurement of a reference quantity $C_{\text {ref }}$ in an identical way and by measuring the offset, $M_{\text {off }}$, by making $m=0$, the parameters $m$ and $M_{\text {off }}$ are eliminated. The final measurement result $P$ is defined as:

$$
P=\frac{M_{\mathrm{ref}}-M_{\mathrm{off}}}{M_{x}-M_{\mathrm{off}}}
$$

In our case, for the sensor capacitance $C_{x}$ it holds that:

$$
C_{x}=\frac{\epsilon A_{x}}{d_{0}+\Delta d}
$$

where $A_{x}$ is the area of the electrode, $d_{0}$ is the initial distance between them, $\epsilon$ is the dielectric constant and $\Delta d$ is the displacement to be measured. For the reference electrodes it holds that:

$$
C_{\text {ref }}=\frac{\epsilon A_{\text {ref }}}{d_{\text {ref }}}
$$

with $A_{\text {ref }}$ the area and $d_{\text {ref }}$ the distance. Substitution of (12) and (13) into (10) and then into (11) yields:

$$
P=\frac{A_{\mathrm{ref}}\left(d_{0}+\Delta d\right)}{A_{x} d_{\mathrm{ref}}}=a_{1} \frac{\Delta d}{d_{\mathrm{ref}}}+a_{0} .
$$


Here, $P$ is a value representing the position while $a_{1}$ and $a_{0}$ are unknown, but stable constants. The constant $a_{1}=$ $A_{\text {ref }} / A_{x}$ is a stable constant provided there is a good mechanical matching between the electrode areas. The constant $a_{0}=\left(A_{\text {ref }} d_{0} /\left(A_{x} d_{\text {ref }}\right)\right.$ will also be a stable constant provided that $d_{0}$ and $d_{\text {ref }}$ are constant. These constants can be determined by a one-time calibration. In many applications this calibration can be omitted; when the displacement sensor is part of a larger system, an overall calibration is required anyway. This overall calibration eliminates the requirement for a separate determination of $a_{1}$ and $a_{0}$.

\section{The Capacitance-to-Period Conversion}

The signals which are proportional to the capacitor values are converted into a period, using a modified Martin oscillator [4] (Fig. 5).

When the voltage swing across the capacitor is equal to that across the resistor and the NAND gates are switched off, this oscillator has a period $T_{\text {off: }}$ :

$$
T_{\text {off }}=4 R C_{\text {off }} \text {. }
$$

Since the value of the resistor is kept constant, the period varies only with the capacitor value. Now, by switching on the right NAND port, the capacitance $C_{x}$ can be connected in parallel to $C_{\text {off }}$. Then the period becomes:

$$
T_{x}=4 R\left(C_{\text {off }}+C_{x}\right)=4 R C_{x}+T_{\text {off }} .
$$

The constants $R$ and $T_{\text {off }}$ are eliminated in the way described in Section IV.

In [2] it is shown that the system is immune for most of the nonidealities of the op amp and the comparator, like slewing, limitations of bandwidth and gain, offset voltages, and input bias currents. These nonidealities only cause additive or multiplicative errors which are eliminated by the three-signal approach.

\section{Vi. Period Measurement with a Microcontroller}

Performing period measurement with a microcontroller is an easy task. In our case, an INTEL 87C51FA is used, which has $8 \mathrm{kByte}$ ROM, 256 Byte RAM, and UART for serial communication, and the capability to measure periods with a $333 \mathrm{~ns}$ resolution. Even though the counters are $16 \mathrm{~b}$ wide, they can easily be extended in the software to $24 \mathrm{~b}$ or more.

The period measurement takes place mostly in the hardware of the microcontroller. Therefore, it is possible to let the CPU of the microcontroller perform other tasks at the same time (Fig. 6). For instance, simultaneously with the measurement of period $T_{x}$, period $T_{\text {ref }}$ and period $T_{\text {off }}$, the relative capacitance with respect to $C_{\text {ref }}$ is calculated according to (11), and the result is transferred through the UART to a personal computer.

\section{EXPERIMENTAL Results}

The sensor is not sensitive to fabrication tolerances of the electrodes. Therefore in our experimental setup we used simple printed circuit board technology to fabricate

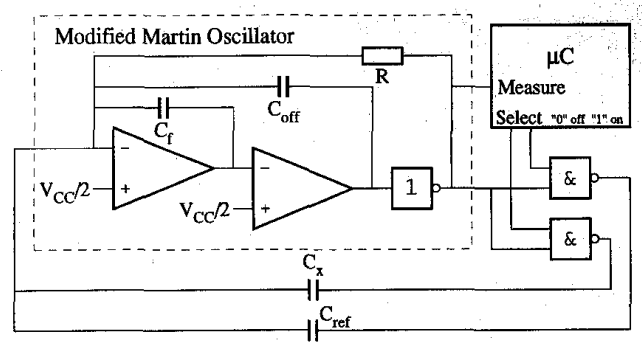

Fig. 5. Modified Martin oscillator with microcontroller and electrodes.

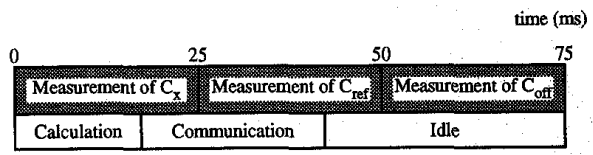

Fig. 6. Period measurement as background process.

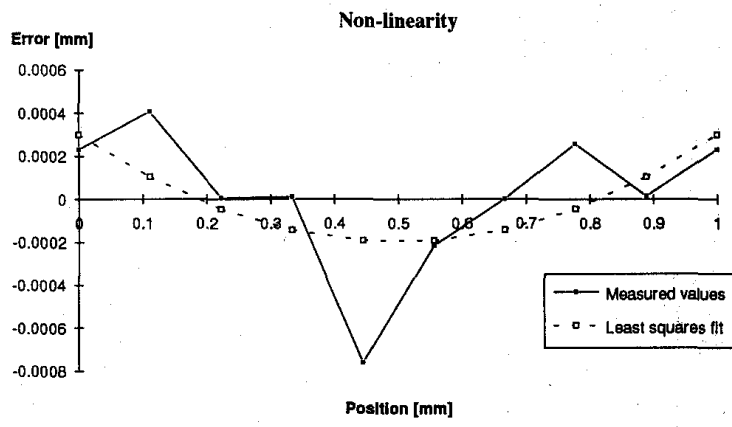

Fig. 7. Position error as function of the position and estimate of the nonlinearity.

the electrodes, which have an effective area of $12 \mathrm{~mm} \times$ $12 \mathrm{~mm}$. The guard electrode has a width of $15 \mathrm{~mm}$, while the distance between the electrodes is about $5 \mathrm{~mm}$. When the distance between the electrodes is varied over a $1 \mathrm{~mm}$ range, the capacitance changes from $0.25 \mathrm{pF}$ to $0.3 \mathrm{pF}$. Thanks to the chosen concept, even a simple dual op amp (TLC272AC) and CMOS NAND's could be used, allowing a single $5 \mathrm{~V}$ supply voltage. The total measurement time amounts to only $100 \mathrm{~ms}$, where the oscillator was running at about $10 \mathrm{kHz}$.

The system was tested in a fully automated setup, using an electrical XY table, the described sensor and a personal computer. To achieve the required measurement accuracy the setup was autozeroed every minute. In this way the nonlinearity, long-term stability and repeatability have bene found to better than $1 \mu \mathrm{m}$ over a range of $1 \mathrm{~mm}$ (Fig. 7). This is comparable to the accuracy and range of the system based on a PSD as described in [2].

As a result of these experiments, it was found that the resolution amounts to approximately $20 \mathrm{aF}$. This result was achieved by averaging over 256 oscillator periods. A further increase of the resolution by lengthening the measurement time is not possible due to the $1 / f$ noise produced by the first stages in both the integrator and the comparator. 
The absolute accuracy can be derived from the position accuracy. Since a $1 \mathrm{~mm}$ displacement corresponds to a change in capacitance of $50 \mathrm{fF}$, the absolute accuracy of $1 \mu \mathrm{m}$ in the position amounts to an absolute accuracy of $50 \mathrm{aF}$.

\section{CONCLUSION}

A low-cost, high-performance displacement sensor has been presented. The system is implemented with simple electrodes, an inexpensive microcontroller and a linear capacitance-to-period converter. When the circuitry is provided with an accurate reference capacitor, the circuit can also be used to replace expensive capacity-measuring systems.

\section{REFERENCES}

[1] G. C. M. Meijer and R. Schrier, "A linear high-performance PSD displacement transducer with a microcontroller interfacing," Sensors and Actuators, A21-A23, pp. 538-543, 1990.

[2] J. van Drecht, G. C. M. Meijer, and P. C. de Jong, "Concepts for the design of smart sensors and smart signal processors and their application to PSD displacement transducers," Digest of Technical Papers, Transducers ' 91 .

[3] W. C. Heerens, "Application of capacitance techniques in sensor design," Phys. E: Sci. Instrum., vol. 19, pp. 897-906, 1986.

[4] K. Martin, "A voltage-controlled switched-capacitor relaxation oscillator," IEEE J., vol. SC-16, pp. 412-413, 1981. 